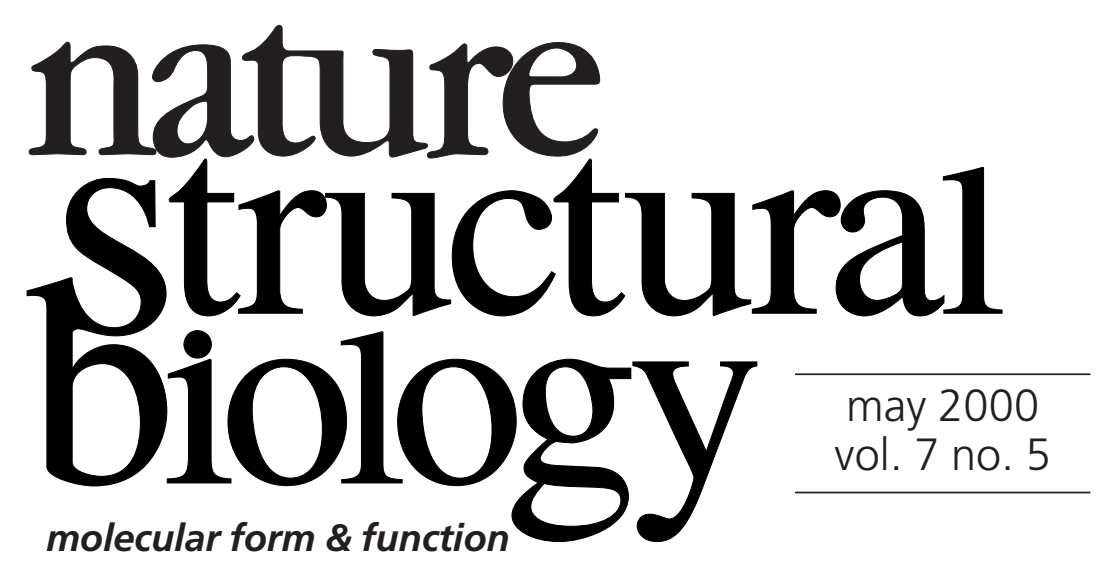

\title{
Brave new world view
}

Last month, $\sim 200$ scientists arrived at the EMBL in Heidelberg, Germany to attend the Millennium Symposium on Structural Biology*. Although the word 'millennium' has been overused recently, this symposium did, in fact, earn the right to use that term, which is synonymous with looking to the future. An underlying theme of the conference was cutting edge technology. Researchers now have a large number of tools at their disposal to analyze biological molecules, and this meeting - more than many others in recent memory - made this apparent. The sessions were organized around some of the most exciting current research in molecular and structural biology: nucleic acid-protein interactions, transport across membranes and within cells, motor proteins and the cytoskeleton, catalysis, and future challenges in structural biology. All were informed by results from a variety of techniques. Here are a few of the highlights.

\section{Big structures and conformational changes}

Francis Tsai (Yale University), one of Paul Sigler's postdoctoral associates, began the first session with a tribute to Sigler, who died earlier this year. His talk focused on how the large eukaryotic initiation complex determines the correct orientation on the DNA, so that transcription proceeds in the proper direction. The same session continued with presentations of some longawaited structures - of prokaryotic core RNA polymerase (Seth Darst, The Rockefeller University) and eukaryotic RNA polymerase II (Patrick Cramer, Stanford University) — both of which should help to answer additional questions about the mechanisms of gene expression. Many similarities appear to exist in the presumed active sites of these complex enzymes, despite the fact that the two complexes interact with many different regulators.

The impression left by the large RNA polymerase structures was quickly supplanted by the effect of another imposing image - that of the $2.7 \AA$ resolution structure of the large $50 \mathrm{~S}$ subunit of the Haloarcula marismortui ribosome, which Tom Steitz (Yale University) presented. This subunit contains $\sim 3,000$ RNA nucleotides, and more than 30 proteins. All of the nucleotides have now been modeled and partially refined, and we can look forward to a detailed description of the protein-RNA interactions in the near future. It is worth noting that since there are currently only 57 protein-RNA complexes listed in the Nucleic Acids Database, this project alone will increase this number by over $50 \%$, once the models of the proteins and the RNA are of sufficient quality for coordinate deposition. Later in the meeting, Ada Yonath (Weizmann Institute) summarized her group's progress on the structures of the large and small ribosomal subunits from Thermus thermophilus, and Joachim Frank (State University of New

${ }^{\star}$ EMBO Conference, Millenium Symposium on Structural Biology at the European Molecular Biology Laboratory in Heidelberg, Germany, March 26-29, 2000. Organized by Andreas Hoenger, Matti Sarraste, Irmi Sinning, and Dietrich Suck.
York at Albany) presented evidence, obtained from cryoelectron microscopy (cryoEM), for conformational changes in the ribosome during the translation cycle.

Together, the results on the ribosome nicely illustrate how various approaches can contribute to the detailed understanding of a biological problem - previous cryoEM data on the whole ribosome have made it easier for crystallographers to interpret their results. In turn, high resolution structures of the ribosomal subunits and their individual components are contributing to an understanding of the conformational changes observed by cryoEM. Other presentations also emphasized a combination of approaches, in particular to elucidate conformational changes. For example, Jonathan Howard (University of Washington), Jon Kull (Max-Planck Institute for Medical Research, Heidelberg), and Ron Milligan (The Scripps Research Institute) discussed 
how kinesin and myosin interact with microtubules or actin polymers, and how these molecular motors might change conformation to generate force. Also, Gabriele Varani showed how coordinated changes seen in an RNA-U1A homodimer structure affect regulation of poly(A) polymerase, a key enzyme in gene expression. In brief, many of the results presented at this meeting can be summed up by two concepts: big structures and conformational changes.

\section{Science future and science fiction}

While there was much inspiring science presented throughout the symposium, some of the most entertaining talks came at the very end. Two of these, by Gerhard Wagner (Harvard University) and Janos Hajdu (Uppsala University), highlighted future challenges in structure determination.

Some context is needed to fully understand a few of the points that Wagner emphasized in his talk. Throughout the meeting, researchers who primarily use NMR in their research had commented informally (and perhaps somewhat enviously) on the large structures that could be solved by crystallography. Some said they were humbled by the big images, since NMR is currently restricted to molecules and complexes of much smaller size. These comments could have left the impression that NMR is far less useful for structure determination. But Wagner successfully countered this in two ways: first by describing improvements in NMR techniques and equipment (such as cryoprobes) that are increasing its capabilities, and second by emphasizing that "big is beautiful, but small is beautiful, too". This statement implies, and rightly so, that structural results on small molecules can be just as intriguing (or in some cases even more intriguing) than results on large supermolecular complexes; a key ingredient to an interesting research story is biological insight. Wagner went on to note that another advantage of using NMR is that it can, relatively easily, provide some degree of insight into both interactions between molecules and conformational changes, two areas that are ripe for further research in all areas of biology.

Discovering how molecules move to perform their functions is a difficult task, since many of the movements have extremely short life times, on the order of picoseconds or femtoseconds. If single molecules could be imaged in this time frame, researchers might be able to visualize important molecular movements. To this end, Janos Hajdu presented what was, admittedly, a science fiction story. His topic: how atomic resolution structures could possibly be determined by X-ray diffraction from one or only a few molecules without crystallization. Theoretically, this may be possible, using short X-ray pulses from a free electron laser. Such radiation is nearly 10 orders of magnitude more intense than what can be obtained from a synchrotron. Hajdu's calculations and computer simulations suggest that data sufficient to construct a three-dimensional image could be collected from a small number of protein molecules in the few femtoseconds before the molecules explode. One major caveat: such an X-ray producing free electron laser does not currently exist. However, research on prototypes is ongoing at the Stanford Linear Accelerator Center (SLAC) in the USA and at Deutsches Elektronen-Synchrotron (DESY) in Hamburg, Germany. Someday, Hajdu might actually be able to put his theories to an experimental test.

\section{Shop talk and take home messages}

The talks generated much lively discussion around the dinner tables each evening. Additionally, particular topics, overheard on several separate occasions, were the subjects of many side conversations. For example, many scientists expressed frustration at the way in which the decision was made about the location of DIAMOND, a new synchrotron planned in the UK. Some believe the decision to build the synchrotron at the Rutherford Appleton Laboratory near Oxford instead of the Daresbury Laboratory near Manchester, the site of the current synchrotron, was made solely for political reasons, without really considering the scientific pros and cons of each location. Another topic of interest to many was structural genomics. Many hope that the money pouring into structural genomics programs around the world is really additional funding, not a redistribution of existing funds from established structural biology research programs. The volatility of biotechnology stocks in the USA, which were fluctuating wildly that week, was also often mentioned. But while such technological issues played a large role in these social conversations, they did not overshadow discussion of the interesting biological results presented throughout the day.

A number of participants remarked that this symposium made it clear that the bar is being raised for both structural and biochemical research: in the future, before a story can be considered suitable for presentation or publication, structural work will require biochemical evidence to support structure-function speculations, and likewise, biochemical and molecular work will require a strong structural context. This is a brave new world view: integration of techniques to solve key biological questions. It was apparent from this meeting that many seem to be taking this on board in their individual research programs — which makes for a promising future. 